\title{
Espacio inducido y territorialización del discurso: Determinando el impacto socioterritorial del imaginario social sobre la inmigración en el área metropolitana de Sevilla*
}

\author{
Ricardo Iglesias Pascual \\ Universidad Pablo de Olavide \\ riglpas@upo.es
}

Recepción: enero de 2015

Aceptación: septiembre de 2015

\section{Resumen}

La creciente complejidad de las dinámicas socioespaciales desarrolladas en el ámbito metropolitano incrementa la importancia de la intersubjetividad espacial a la hora de comprender globalmente el hecho urbano. Así, una de las variables que mayor impacto tiene sobre el imaginario social es la inmigración. Para su comprensión, es necesario realizar un enfoque analítico que aborde holísticamente la dimensión socioespacial intersubjetiva del imaginario sobre la inmigración y su papel en la ciudad. Por ello, proponemos, en el presente artículo, el concepto de espacio inducido y, en concreto, el desarrollo de una propuesta metodológica basada en el análisis del discurso, orientada a determinar el papel del imaginario social metropolitano sobre inmigración, en los procesos de segregación residencial de aquellos barrios que albergan altos porcentajes de población extranjera. Todo ello, desarrollado en el área metropolitana de Sevilla, ha mostrado cómo el espacio inducido desempeña un papel crucial en la realización de los procesos de segregación y estigmatización socioterritorial de aquellos ámbitos percibidos socialmente como "barrios de inmigrantes».

Palabras clave: espacio intersubjetivo; espacio inducido; territorialización del discurso; grupos de discusión; escala metropolitana.

* Este artículo sintetiza la metodología desarrollada en la tesis doctoral El papel de las concepciones espaciales subjetivas en la segregación residencial de los barrios con alta densidad de inmigración: El caso de la ciudad de Sevilla, defendida en julio de 2014 en la Universidad Pablo de Olavide, Sevilla. A su vez, dicho estudio se desarrolló en el marco del proyecto Movilidad y Reconfiguración Urbana y Metropolitana (MOVITRA III), financiado por el Ministerio de Economía y Competitividad. Plan Nacional de I+D+i (CSO2011-29943-C03). 
Resum. Espai induït i territorialització del discurs: Determinant l'impacte socioeconòmic territorial de l'imaginari social sobre la immigració a l'àrea metropolitana de Sevilla

La creixent complexitat de les dinàmiques socioespacials desenvolupades en l'àmbit metropolità incrementa la importància de la intersubjectivitat espacial a l'hora de comprendre globalment el fet urbà. Així, una de les variables que exerceix més impacte sobre l'imaginari social és la immigració. Per comprendre aquest fenomen, és necessari que es desenvolupi un enfocament analític que abordi holísticament la dimensió socioespacial intersubjectiva de l'imaginari sobre la immigració i el paper que té a la ciutat. Per això, proposem, en el present article, el concepte d'espai induït $i$, en concret, el desenvolupament d'una proposta metodològica basada en l'anàlisi del discurs, orientada a determinar el paper de l'imaginari social metropolità sobre immigració, en els processos de segregació residencial d'aquells barris que alberguen alts percentatges de població estrangera. Tot això, desenvolupat a l'àrea metropolitana de Sevilla, ha mostrat com l'espai induït realitza un paper crucial en el desenvolupament dels processos de segregació i estigmatització socioterritorial d'aquells àmbits percebuts socialment com a "barris d'immigrants".

Paraules clau: espai intersubjectiu; espai induït; territorialització del discurs; grups de discussió; escala metropolitana.

Résumé. Espace induit et territorialisation du discours: Déterminer l'impact socio-territorial de l'imaginaire social de l'immigration dans la région métropolitaine de Séville

La compréhension du fait urbain passe de plus en plus par la prise en considération de l'intersubjectivité spatiale du fait de la complexité grandissante des dynamiques sociospatiales métropolitaines. Aussi, l'une des variables qui a le plus d'impact sur l'imaginaire social est l'immigration. Pour faciliter sa compréhension, il est essentiel de développer une approche analytique qui aborde de manière globale la dimension socio-spatiale intersubjective de l'imaginaire sur l'immigration et son rôle dans la ville. Dans le cadre de cet article, la notion d'espace induit et le développement d'une méthodologie fondée sur l'analyse du discours permettent de déterminer le rôle de l'imaginaire social métropolitain sur l'immigration dans les processus de ségrégation résidentielle dans les quartiers à forte concentration d'étrangers. Cette étude porte sur la région métropolitaine de Séville, où l'on démontre que l'espace induit jour un rôle crucial dans le développement des processus de ségrégation et de stigmatisation socio territoriale de ces zones socialement percues comme des "quartiers immigrés".

Mots-clés: espace intersubjectif; espace d'induit; territorialisation de la parole; groupes de discussion; échelle métropolitaine.

Abstract. Induced space and the territorialization of discourse: Determining the socio-territorial impact of social imaginary about immigration in the metropolitan area of Seville

The growing complexity of the socio-spatial dynamics of metropolitan spaces increases the importance of spatial intersubjectivity when attempting to gain a global understanding of urban reality. One of the variables that has the greatest impact on the social imaginary is immigration. To understand this phenomenon, it is necessary to develop an analytical approach that holistically explores the intersubjective socio-spatial dimension of the imaginary about immigration and its role in the city. To do so, we propose the concept of induced space and a discourse analysis-based methodology aimed at determining the role of the metropolitan social imaginary about immigration in processes of residential segregation in neighborhoods with a high percentage of foreigners. We show how, in the metropolitan 
area of Seville, the induced space plays a key role in processes of socio-territorial segregation and stigmatization of areas socially perceived as "immigrant neighborhoods".

Keywords: intersubjective space; induced space; territorialization of discourse; focus group; metropolitan scale.

\section{Sumario}

1. A modo de introducción y contextualización epistemológica

2. El espacio inducido. La subjetividad espacial a la escala metropolitana

3. Bases metodológicas de la territorialización del discurso
4. Desarrollando la territorialización del discurso: el trabajo de campo

5. Consideraciones finales sobre el espacio inducido y el impacto socioterritorial del imaginario sobre la inmigración

Referencias bibliográficas

\section{A modo de introducción y contextualización epistemológica}

El ejercicio de sintetizar el modelo metodológico desarrollado en una investigación siempre es una tarea compleja, ya que supone concretar un aspecto central de la misma, en cuya elección se plasman gran parte de los principios epistemólogicos y prácticos puestos en marcha. En este caso, el carácter holístico y transdisciplinario del objeto de estudio abordado y de la metodología que aquí se muestran dificultan aún más este trabajo de síntesis. A pesar de ello, consideramos que puede ser interesante para el lector reflexionar sobre la pertinencia de la presente propuesta metodológica y el tratamiento teórico llevado a cabo a la hora de analizar el espacio subjetivo en su dimensión metropolitana y socialmente colectiva. Con el objeto de no dispersarnos en el tratamiento de una cuestión tan amplia como la intersubjetividad espacial, hemos centrado el análisis en el papel que el imaginario social desarrollado sobre el extranjero presenta en relación con los procesos de segregación y estigmatización territorial de los barrios que acogen altos porcentajes de dicha población.

Para llevar a cabo la investigación, se decidió seleccionar el área metropolitana de Sevilla como unidad territorial de estudio, a pesar de la escasa población extranjera que paradójicamente alberga ${ }^{1}$. A pesar de ello, la localización concentrada de dicha población en ámbitos barriales ${ }^{2}$ muy concretos y el carácter por tanto singular de estos espacios en relación con el resto de la zona metropolitana,

1. La población extranjera en el municipio de Sevilla suponía el 5,4\% en 2011, momento en el que se realiza el trabajo de campo, y el 5,1\% según el Padrón Continuo de Población de 2014. Dichos porcentajes representan unos escasísimos valores en comparación con municipios como Madrid (13,19\%) o Barcelona (17,04\%) en 2014.

2. De hecho, presenta valores de segregación residencial de la población extranjera superiores, en algunos casos, a ciudades con mayor presencia de extranjeros como Madrid o Barcelona. Para más información, véase Palacios y Vidal (2014). 
permitió visualizar con gran claridad los discursos construidos sobre aquellos ámbitos catalogados socialmente como "barrios de inmigrantes» ${ }^{3}$.

Tradicionalmente, el espacio subjetivo ha sido concebido como una dimensión vivida y experimentada de forma diferente por sus habitantes, según símbolos y valores que se van elaborando a través de mecanismos fisiológicos y psicológicos de percepción y la experiencia personal. Es decir, se ha abordado el estudio del espacio conocido y aprehendido individualmente (Vilá Valentí, 1983). Este enfoque se ha apoyado normalmente sobre la imagen mental como eslabón que existe entre el medio real y la conducta, así como sobre el principio de racionalidad limitada que rige el comportamiento del hombre. De este modo, el espacio percibido por medio de todos los sentidos adquiere una significación concreta para quienes viven inmersos en él, originada a partir de la experiencia continua y cotidiana de la vida que allí tiene lugar. Metodológicamente, los estudios sobre la percepción se han basado en la realización de cuestionarios individuales, mapas mentales o la realización de itinerarios, pero siempre centrados en una dimensión individual de recogida de la información. Después se realiza una agregación de datos para recrear la subjetividad colectiva. A su vez, siempre se han orientado estos recursos al análisis del espacio conocido y familiar del entrevistado.

Recientemente, al análisis sobre el espacio subjetivo, se puede añadir la aportación realizada desde disciplinas como la sociología y la antropología, con el estudio de los imaginarios sociales y, más concretamente, de los imaginarios urbanos (García Canclini, 1996, 1997; Lindón et al., 2006). Bajo el término imaginarios sociales, se engloban todas aquellas representaciones colectivas que rigen los sistemas de identificación y de integración social y que hacen visible lo implícito u oculto socialmente (Pintos, 1995). Lo imaginario posibilita recrear unas referencias semejantes de percepción (espaciales, temporales, geográficas, etc.), de explicación (marcos lógicos, emocionales, etc.) y de intervención (estrategias, programas, políticas, etc.). El estudio del imaginario urbano se ha centrado en analizar los discursos y las representaciones que el ciudadano hace de la ciudad y de las relaciones que en ella se dan, ofreciendo una visión más holística que la generada tradicionalmente por el mero análisis del espacio percibido. Al igual que los estudios sobre percepciones, metodológicamente, el análisis de los imaginarios se ha basado en la generación de datos primarios mediante el desarrollo de registros individuales (Silva, 1992).

Sin embargo, el presente artículo, partiendo de los enfoques hasta ahora abordados, se centra en exponer el desarrollo de una propuesta metodológica, a la que hemos denominado territorialización del discurso, orientada hacia el estudio de la subjetividad espacial a escala metropolitana en su dimensión intersubjetiva. Es decir, una propuesta que permita analizar la subjetividad recreada sobre un espacio urbano cada vez de mayor extensión, recogiendo su dimensión colectiva más allá de la mera agregación de datos individuales.

3. Para una visión más detallada acerca de los criterios utilizados a la hora de establecer estas zonas, véase Iglesias Pascual (2015). 
Por tanto, el objetivo principal del artículo es mostrar la validez y las limitaciones principales derivadas de la aplicación de unos instrumentos metodológicos orientados hacia el análisis de la intersubjetividad espacial, recreada por los ciudadanos y su incidencia en los procesos de segregación residencial. Para ello, primero se desarrolla un análisis sobre las características de la subjetividad espacial a escala metropolitana, a fin de entender los instrumentos necesarios para su estudio. En segundo lugar, se abordan los principios metodológicos inspiradores de la investigación y los instrumentos concretos en los que estos se han plasmado. Posteriormente, se describe el desarrollo metodológico mediante el trabajo de campo, reflexionando finalmente, a la luz de los resultados principales, sobre las potencialidades y las aristas de la territorialización del discurso para el estudio de la subjetividad espacial y la incidencia del imaginario social sobre los procesos de segregación de la población extranjera.

\section{El espacio inducido. La subjetividad espacial a la escala metropolitana}

La gran variedad de enfoques que abordan la dimensión subjetiva del espacio y la dificultad de discernir entre las diferentes dimensiones del mismo resulta evidente al consultar la bibliografía disponible. Muestra de ello, a su vez, es la habitual equiparación de lo percibido con lo imaginado como si fuesen elementos intercambiables. Así, autores tan diversos como Lynch (1960), Lowenthal (1961) o Tuan (1974) ponen de manifiesto la estrecha relación existente entre el espacio percibido e imaginado, entre las percepciones que los ciudadanos construyen y los imaginarios que recrean posteriormente a partir del espacio vivido. De hecho, suele plantearse la construcción del espacio subjetivo como consecuencia de una percepción espacial previa. De este modo ha sido tratado tradicionalmente desde la psicología ambiental, la sociología, la antropología y la geografía.

Sin embargo, en las áreas metropolitanas, ante la creciente dificultad de conocer el territorio en su totalidad ${ }^{4}$, este es reconstruido por los ciudadanos a través de informaciones recogidas a diario mediante las interrelaciones sociales, la movilidad cotidiana o la información recibida de los medios de comunicación ${ }^{5}$ (García Canclini, 1996, 1997). Ante un espacio urbano que crece de forma constante más allá de la ciudad consolidada, resulta cada vez más complejo para el sujeto conocer completamente todo el territorio que abarca su ciudad. Partiendo de estas características de la realidad urbana, se ha decidido centrar el artículo sobre la construcción del espacio subjetivo como dimensión socioespacial imaginada, desarrollada sin una percepción o una experiencia personal previa. Se trata de focalizar el análisis en la dimensión

4. Diferentes estudios urbanos y metropolitanos (Hall, 1996; Indovina, 1998; Monclús, 1998; Dematteis, 1998; Feria, 2013) ponen de manifiesto el carácter cada vez más extenso y disperso de la ciudad sobre el territorio.

5. Del mismo modo, la población forma su visión sobre aspectos como la inmigración (Van Dijk, 2003; Igartua et al., 2005, 2006; Álvarez Gálvez, 2011) o la delincuencia (Echazarra, 2014). 
territorial urbana recreada sin un conocimiento directo por parte del sujeto o del grupo social. Esta dimensión del espacio imaginado adquiere cada vez una mayor importancia ante el tamaño del territorio metropolitano actual, que dificulta la construcción de un conocimiento completo de la ciudad y otorga, por tanto, una mayor importancia al imaginario en el día a día del ciudadano.

Tal como indica García Canclini (1996), la mayor complejidad y la gran extensión del espacio metropolitano plantean un incremento del papel de los medios de comunicación en la formación y en la divulgación de los imaginarios urbanos. En este sentido, cabe reseñar cómo los medios ofrecen elementos para recrear la ciudad, pero no contribuyen a enriquecer su visión ni su conocimiento (Aguilar, 2006). Pese al énfasis en la novedad, los medios suelen concentrarse en lo conocido, prolongando estereotipos formados históricamente y que refuerzan los imaginarios sociales ya existentes.

A este papel de los medios, se debe añadir, del mismo modo, la importante influencia que ejerce en la construcción del imaginario la información que el ciudadano extrae de su interrelación social cotidiana, la cual incide en el mantenimiento y en la expansión de aspectos estereotipados (Iglesias Pascual, $2014 b$ ) y que, en gran medida, intervienen en desarrollar la denominada por Wacquant (2007a y 2007b) estigmatización territorial, especialmente en aquellas zonas de la ciudad caracterizadas por la marginalidad o la presencia de población extranjera de bajo nivel de renta. Dicha estigmatización se plasma en diferentes imaginarios compuestos por estereotipos sociales que generan zonas de topofilias y topofobias en la ciudad, tal como indicaba Tuan (1974).

Partiendo de todos estos principios epistemológicos, en el presente artículo, se propone afrontar el estudio de la subjetividad espacial a nivel metropolitano a través de lo que hemos denominado concepciones espaciales subjetivas (CES). Bajo dicho constructo, integramos toda la amplia variedad de dimensiones que estudian la subjetividad espacial (Iglesias Pascual, 2014a). Los enfoques anteriormente referidos, conformados básicamente mediante las diferentes categorías analíticas en las que el investigador clasifica los elementos que integran el espacio vivido - ya sea en su dimensión perceptiva, imaginaria o simbólica-, se centran normalmente en el análisis de uno solo de estos aspectos. Sin embargo, con las CES, se propone analizar la construcción de la intersubjetividad socioespacial a partir del punto de vista del sujeto. Con esto se plantea como este, en su proceso de reconstrucción del espacio, no discrimina entre diferentes categorías analíticas, como haría el investigador. Es decir, la construcción de la subjetividad espacial se trata de un proceso complejo, donde interviene lo percibido, lo imaginado, lo simbólico, etc., generando con todo ello una visión compuesta por elementos individuales e intersubjetivos, tanto de su entorno urbano habitual como de aquel en el que interactúa ocasionalmente. Con dicha información, se construye el imaginario sobre los diferentes ámbitos de la ciudad, el cual, a su vez, queda plasmado en un discurso social conformado como el elemento que explícita dicho imaginario colectiva y públicamente. De aquí se deduce la posición central que el artículo otorga al análisis del discurso como elemento definidor de las mencionadas CES. 
En el ámbito metropolitano, adquieren una importancia especial aquellas CES construidas sobre los espacios desconocidos o ignotos para el individuo, cuya visión de los mismos se basa en la información transmitida por los medios o mediante diferentes tipos de interrelaciones sociales, pero sin conocer directamente el ámbito territorial sobre el que se originan. Estas concepciones desarrollarían lo que hemos determinado denominar espacio inducido (Iglesias Pascual, 2014a). Con dicho término, englobamos toda aquella subjetividad espacial desarrollada sobre ámbitos poco conocidos o completamente desconocidos, fruto de la influencia tanto de los medios de comunicación como de las interrelaciones sociales cotidianas, los imaginarios sociales y los discursos de ellos derivados a nivel individual y social. Se ha denominado inducido puesto que, en su formación, intervienen fundamentalmente variables externas a la experiencia cotidiana del individuo o del grupo, sin que haya un conocimiento directo del territorio sobre el que se construye, por lo que, propiamente, no se le puede considerar imaginado.

Este espacio inducido no es un constructo ex nibilo, fruto de una generación espontánea, que se origine sin influencia externa. Se trata de una dimensión espacial interrelacionada indirectamente con el espacio vivido por el individuo, es decir, las vivencias del entorno cotidiano inciden en el modo en que se construye la imagen sobre el resto de la ciudad. La imagen que se recrea sobre los diferentes ámbitos de la ciudad se encuentra determinada por las

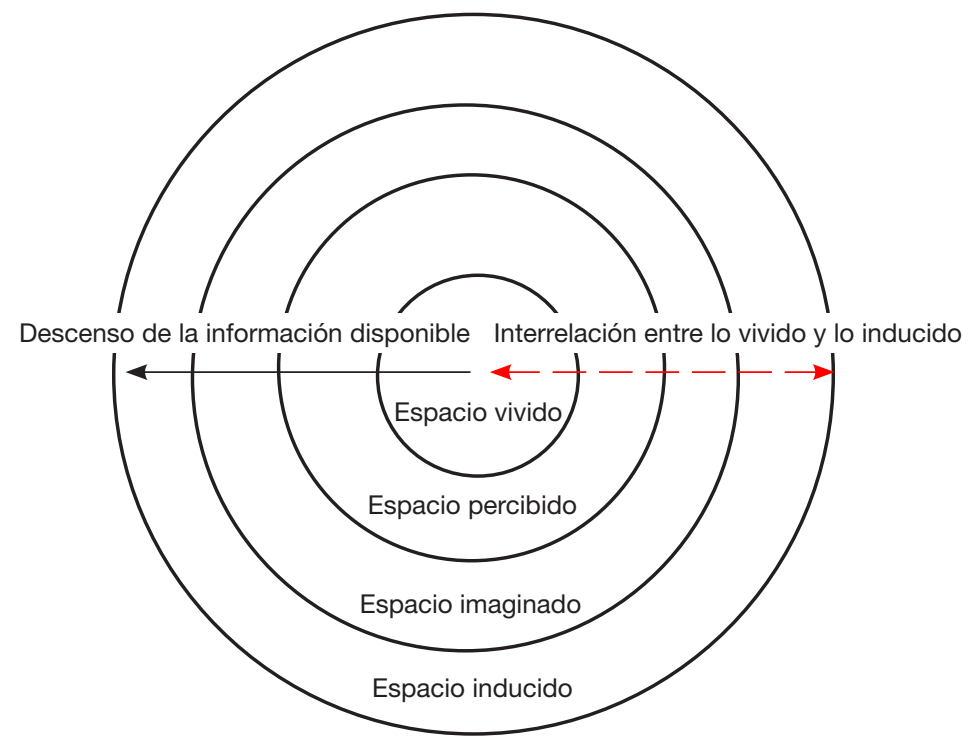

Figura 1. Formación e interrelaciones del espacio inducido.

Fuente: elaboración propia. 
características del entorno barrial propio. En este sentido, el entorno concreto determina nuestra manera individual y colectiva de recrear los espacios simbólicos, del ocio, del miedo, etc., en nuestro imaginario social. A su vez, dicho espacio inducido, construido individual y colectivamente, desempeña un papel crucial a la hora explicar los comportamientos espaciales dentro del espacio vivido (figura 1). Desde este punto de vista, la propuesta epistemológica del espacio inducido puede representar una aportación a la hora de entender el comportamiento socioespacial, ante la cada vez mayor frecuencia de los espacios ignotos en el difuso y extenso territorio metropolitano.

\section{Bases metodológicas de la territorialización del discurso ${ }^{6}$}

La complejidad y la amplitud del objeto de estudio propuesto, junto a la importancia de adentrarse en cuestiones íntimamente relacionadas con aspectos intersubjetivos, han sido factores clave para decantarse por la utilización de una metodología con un carácter lo más holístico posible. Solo mediante un enfoque globalizador e integrador se puede analizar la gran cantidad de variables asociadas al tema. Partiendo de esta premisa, hemos considerado que una metodología básicamente cualitativa resulta la más adecuada a la hora de captar los discursos de la población seleccionada y sus mecanismos de construcción social de la realidad (Berger y Luckmann, 1966). En este sentido, se busca indagar sobre el papel de las experiencias y de las interrelaciones personales, así como sobre la importancia del lugar en el espacio social y urbano para la construcción de dichas subjetividades (Relph, 1976; Tuan, 1974; Certeau, 1980; Augé, 1992), sin olvidar el creciente papel de los medios de comunicación (Van Dijk, 2003; Cea D’Ancona, 2004; Igartua et al., 2005).

Este mayor peso otorgado a los instrumentos cualitativos se basa en la experiencia de otras investigaciones que han mostrado cómo los cuestionarios de carácter cuantitativo pueden no recoger adecuadamente todas las intencionalidades de aquellos individuos que presenten un perfil reacio a la inmigración. Muestra de ello, según Cea D’Ancona (2009b), es que las personas de mayor nivel educativo son las más proclives a decantarse por un mayor sesgo de deseabilidad social en sus discursos y respuestas, ya que, al percibir la intencionalidad de la pregunta, procuran no mostrar actitudes racistas o xenófobas, especialmente cuando han de responder ante un entrevistador (Krysan, 1998). Con la utilización de estrategias de carácter cualitativo, se facilita la generación de un contexto abierto donde el entrevistado, al hablar libremente, muestra mediante su discurso aspectos implícitos en sus actitudes que con el corsé de un cuestionario cerrado no suelen aflorar.

Sobre esta base, se ha considerado que, para captar las características de las concepciones espaciales individuales y colectivas que intervienen en la construcción del imaginario sobre la inmigración, una metodología cualitativa per-

6. Con esta metodología, no se pretende alcanzar generalizaciones estadísticas extrapolables a otras ciudades o regiones con alta densidad de población inmigrante. 
mite adquirir una mayor flexibilidad de respuesta y participación, mostrando más validez a la hora de recoger información tanto de lo explicito como de lo implícito del discurso social. La intención principal es hacer reflexionar a los ciudadanos de manera general sobre la ciudad, sobre los espacios que conocen, sobre aquellos ámbitos de los que solo tienen referencias parciales y, por último, sobre las zonas que, básicamente, imaginan o recrean. Únicamente teniendo en cuenta la doble dimensión de lo urbano, como espacio objetivo y subjetivo, tal como es imaginado y percibido por sus habitantes, se alcanza un análisis holístico y global propio de la Geografía. De acuerdo con estas consideraciones, resulta evidente que los principios básicos inspiradores de esta propuesta metodológica se basan en la idea de la sociedad como realidad subjetiva que explica de manera determinante el modo de vivir, percibir e imaginar el espacio socialmente.

A partir de ello, a la hora de determinar los principales instrumentos metodológicos aplicados en la investigación, se ha tenido en cuenta el carácter especialmente implícito de la realidad a analizar. La dimensión de la intersubjetividad social se mueve entre los sentimientos y las creencias latentes en los individuos, por lo que los instrumentos necesarios para hacerlos aflorar se deben caracterizar por su capacidad para indagar en lo colectivo, lo simbólico y, en ocasiones, lo oculto de las construcciones sociales. Para una mayor claridad expositiva en la descripción de las estrategias metodológicas desarrolladas, se han dividido los instrumentos metodológicos utilizados en dos categorías. En primer lugar, se han establecido aquellos destinados a la recogida de información para posteriormente centrarnos en aquellos utilizados en el análisis y en la interpretación del discurso.

\subsection{Generando los registros discursivos}

\section{Los grupos de discusión}

Tal como indica Alonso (1996), el grupo de discusión (a partir de ahora GD) es, fundamentalmente, un proyecto de conversación socializada en el que la producción de una situación de comunicación grupal sirve para la captación y el análisis de los discursos ideológicos y de las representaciones simbólicas que se asocian a cualquier fenómeno social. Estas representaciones sociales planteadas como formas de conocimiento, colectivamente elaboradas y compartidas, determinan la forma común en la que los diferentes grupos humanos construyen e interpretan su realidad y la de otros colectivos. Debido a estas características, la dinámica grupal y los discursos derivados se han planteado como un elemento principal para afrontar el estudio de las CES, el espacio inducido y los elementos que actúan en la formación de ambas dimensiones.

\section{La entrevista individual}

A la hora de decidir qué tipo de entrevista se debe plantear en la investigación, se ha optado por utilizar el formato de entrevista semiestructurada, con el objeto de, sin caer en la estructura rígida de un cuestionario cerrado, poder orientar 
con facilidad la conversación hacia aquellos aspectos sobre los que se deseaba obtener información. Con esta premisa se establecieron guiones orientativos que, de una manera flexible, articulan la entrevista. Según Corbetta (2007), el guión flexible de la entrevista se determina para poder establecer un perímetro dentro del cual el entrevistador decide no tanto el orden y la formulación de las preguntas, como el grado de profundización en algún tema y, en su caso, en cuál de ellos.

\section{La observación participante}

La necesidad de captar la dimensión implícita de los discursos generados sobre la inmigración y los barrios en los diferentes GD llevó a la decisión de utilizar la observación y la toma de notas una vez que los participantes pensaban que, acabada la grabación, no eran recogidas sus opiniones. Esto resultó especialmente importante ante la actitud generalizada de los participantes tras finalizar la dinámica, de mostrar tonos y matices sustancialmente distintos a los utilizados durante la misma. De hecho, tal como ha reflejado Cea D’Ancona (2004, 2005, 2009a), la tendencia a recrear discursos buscando la mayor deseabilidad social posible a la hora de hablar sobre la inmigración, es uno de los principales problemas que se le plantean en la investigación social. Por otro lado, aunque no se debe olvidar que la subjetividad y el carácter individual de los testimonios dificultan la generalización de sus observaciones, por lo que no pueden ser considerados discursos extrapolables o generalizables, sí resultan elementos sumamente esclarecedores de la dimensión implícita de las actitudes hacia la inmigración.

\section{La fotoelucidación}

Autores como Ruiz (2009) o Conde (2009) remarcan la importancia de impulsar el diálogo en las dinámicas grupales para que empiecen a discurrir los discursos. A la hora de iniciar las dinámicas, se ha decidido que lo más motivador para ello era la técnica de la fotoelucidación. Se trata de una técnica cualitativa basada en el uso de la imagen como estímulo de la conversación entre la persona que entrevista y la que es entrevistada ${ }^{7}$. Dicho recurso resulta especialmente efectivo a la hora de activar los mecanismos de la memoria y las diferentes sensibilidades que permiten mostrar tanto la dimensión explícita como implícita del discurso. La inclusión de imágenes busca facilitar la comunicación y evitar la extrañeza inicial de la situación de entrevista (Hurworth, 2003). La utilización de la fotoelucidación en esta investigación se ha plasmado en la visualización, previa al inicio de la dinámica grupal, de un vídeo de 12 minutos de duración, en el que se observaban escenas de diferentes barrios de la ciudad ${ }^{8}$. Con un tratamiento similar, se muestra a los participantes tanto

7. Ha sido utilizada para el estudio de los imaginarios urbanos de Ciudad de Méjico en García Canclini (1996).

8. Las escenas del vídeo fueron tomadas a lo largo del mes de mayo de 2013 en distintos puntos de la ciudad, siempre en la misma hora del día. 
espacios en los que se centra la investigación, como puntos nodales de la ciudad relacionados con el transporte, los servicios comerciales y las zonas caracterizadas por su función residencial.

\subsection{El discurso y su análisis}

Desde un punto de vista sociológico, se puede definir la palabra discurso como cualquier práctica por la que los sujetos dotan a la realidad de sentido. El interés por el discurso para el conocimiento de la realidad social parte de la consideración del carácter subjetivo de la acción social. Dicho carácter subjetivo estaría orientado por el sentido que da el sujeto a su propia acción, por lo que es preciso atender a ello para su comprensión y su explicación. Ahora bien, los principios por los que los sujetos orientan su acción son, en buena medida, producidos y compartidos socialmente. De este modo, hay que tener en cuenta el punto de vista del sujeto como base para la explicación de la acción social, pero sin olvidar la importancia de la intersubjetividad como característica esencial de la realidad social (Schutz, 1972). Siendo la comunicación solo posible en el marco de una cierta intersubjetividad, sus productos, es decir los discursos, contienen dicha intersubjetividad de manera implícita. En nuestro caso, considerando que las CES son productos de la intersubjetividad social, el análisis del discurso se convierte en fundamental a la hora de comprender el papel de la subjetividad espacial, su influencia en el comportamiento en el espacio urbano y, más concretamente en este caso, sobre la segregación residencial de la población extranjera.

\section{Niveles de análisis del discurso en la investigación territorial}

A la hora de abordar el análisis del discurso, en primer lugar, se deben tener en cuenta las diferentes tipologías de análisis posibles, así como sus distintas dimensiones y corrientes. Son diversos los niveles de análisis que se pueden plantear, desde la clasificación realizada por Ibáñez (1979), pasando por la de Ortí (1986) o la más reciente de Alonso (1998), sintetizada y simplificada a grandes rasgos por Ruiz (2009).

Según este último autor, se pueden determinar tres niveles de análisis del discurso: textual, contextual e interpretativo. El análisis textual y el contextual formarían parte del análisis sociológico del discurso, pero no son en sí mismos análisis sociológicos. El nivel textual ofrece una caracterización del discurso, centrada fundamentalmente en el plano del enunciado. El análisis contextual considera el discurso en su dimensión de hecho o acontecimiento singular. La interpretación, por último, proporciona una explicación del discurso centrada en el plano sociológico, considerando el discurso en sus dimensiones informativa, ideológica o bien de producto social. Es justamente este nivel interpretativo el elemento distintivo del análisis sociológico.

En la presente propuesta de territorialización del discurso, no se plantea tanto la profundización en el análisis sociológico, como la determinación de las principales categorías temáticas que se implican en la formación de las 
CES. Esto no es óbice para que, puntualmente, se haga un acercamiento al análisis sociológico de las principales categorías del discurso, de cara a una mejor comprensión de la formación de las CES y su variación en el espacio metropolitano.

A la hora de conformar la tipología de análisis discursivo llevado a cabo, se ha tenido en cuenta el enfoque desarrollado por Conde (2007) en modelos de investigación con un componente territorial similar al aquí propuesto. Conde propone el análisis discursivo desde un enfoque que no se ciñe estrictamente al análisis sociológico. Se trataría de un análisis discursivo basado en una interpretación de los textos de una manera más libre, de modo que la aportación de los verbatims de los diferentes GD ocuparía una función crucial como exponente de las posiciones intersubjetivas establecidas socialmente.

En este sentido, el análisis del discurso desarrollado se ha centrado en el estudio temático de las principales categorías discursivas que se desarrollan intersubjetivamente desde el espacio metropolitano respecto a la figura del inmigrante extranjero y los barrios donde se asientan. De este modo, mediante la reconstrucción de los discursos, se consigue una reproducción de las cogniciones socialmente establecidas en la ciudad respecto a los barrios con mayor porcentaje de población extranjera residente. En esta misma línea lo plantea el colectivo IOÉ (2010), mostrando cómo, en numerosos estudios sobre la inmigración que utilizan y analizan material grupal, son los investigadores quienes seleccionan, ordenan y presentan el material, adoptando un enfoque situado claramente en el nivel de las opiniones manifiestas. Una estrategia similar desarrollan Cea D'Ancona y Valles (2010), utilizando el material grupal para

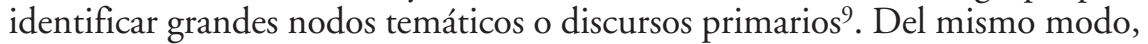
Rinken y Pérez Yruela (2007) plantean su análisis sobre las opiniones de los andaluces respecto a la inmigración.

Por tanto, se puede considerar que el análisis discursivo desarrollado en los GD realizados durante la investigación estaría básicamente dentro del nivel textual del discurso. Partiendo de esta consideración, se han establecido tres premisas previas que organizan el análisis desarrollado para determinar las principales categorías discursivas.

En primer lugar, se han diferenciado tres grandes ámbitos territoriales para el análisis: la ciudad central y la primera y la segunda coronas del área metropolitana de Sevilla. Con dicha división territorial, se pretende contrastar la evolución que muestran los discursos en su transmisión y transformación por el territorio metropolitano. En segundo lugar, se enfoca el estudio de las diferentes categorías observables en estos tres ámbitos, atendiendo al nivel de transversalidad territorial ${ }^{10}$ del discurso y a la influencia del estatus socioeconómico en el mismo. Estas dos variables se situarían dentro de un nivel textual y

9. Véase <http://explotacion.mtin.gob.es/oberaxe/documentacion_descargaFichero. action?bibliotecaDatold $=166>$.

10. Por transversalidad territorial, se hace referencia a la mayor o menor capacidad de determinados discursos a expandirse por todo el territorio metropolitano. 


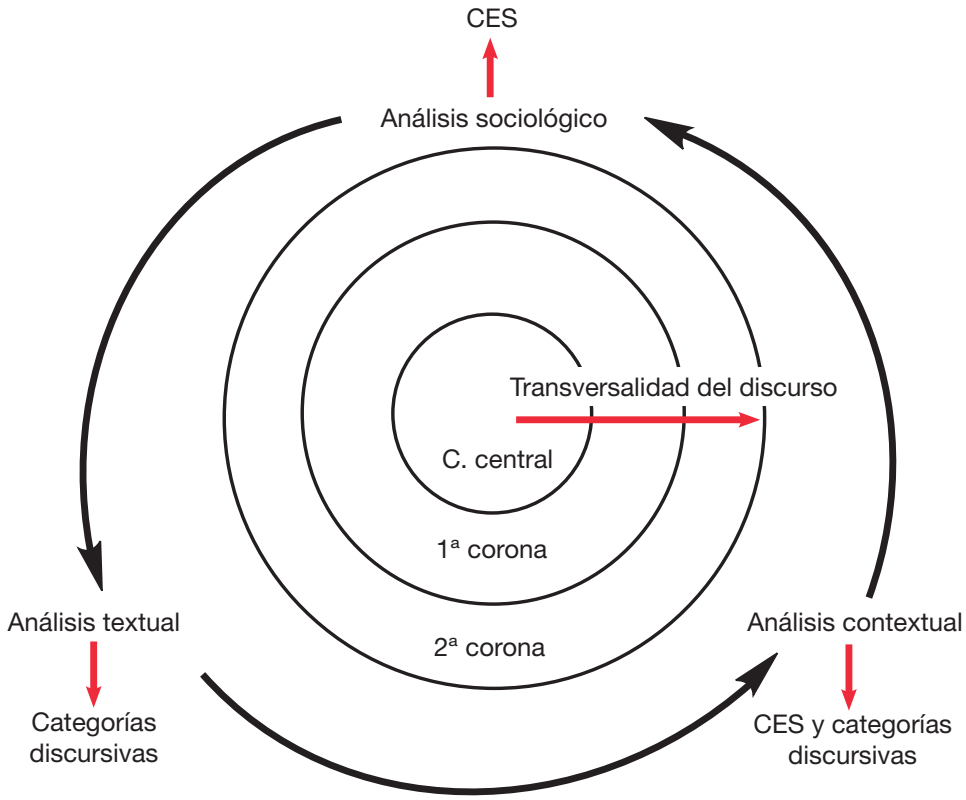

Figura 2. Niveles de análisis discursivo y territorialización del discurso.

Fuente: elaboración propia.

contextual del análisis del discurso. Por último, con un carácter más interpretativo, cercano al análisis sociológico, estaría la determinación de las propias CES, planteada en un plano de mayor abstracción y generalización a partir de las principales posiciones discursivas detectadas en torno a la inmigración y a los barrios donde se concentra.

Atendiendo a la organización del análisis del discurso propuesto (figura 2), resulta evidente la interrelación existente, tanto entre los tres niveles de análisis del discurso como entre las diferentes conceptualizaciones extraídas de dicho análisis. Aunque el proceso aparezca de una manera escalonada, en cada estadío del mismo hay una intervención de los diferentes niveles de análisis que han permitido llegar a la determinación final de cuáles son las principales CES que se desarrollan sobre los barrios estudiados, cómo se generan y se transforman en el espacio metropolitano, así como su reflejo en los comportamientos sociales y, por ende, en la movilidad residencial que acaba generando la segregación de la población inmigrante.

\section{Desarrollando la territorialización del discurso: el trabajo de campo}

Para comprender los motivos implícitos en el diseño del trabajo de campo, se debe tener en cuenta que partimos de la consideración de las CES como 
constructo social colectivo que determina en gran medida las actitudes que los ciudadanos desarrollan hacia determinados ámbitos de la ciudad. De igual modo, el planteamiento del estudio a escala metropolitana está motivado por la importancia que presenta esta dimensión territorial ${ }^{11}$ y el interés en analizar de qué modo se transforma la subjetividad espacial según el lugar del espacio metropolitano donde se resida.

Como se expuso en el apartado introductorio, esta propuesta metodológica se ha desarrollado para analizar el papel de la subjetividad espacial en el proceso de segregación residencial de aquellos barrios de la ciudad de Sevilla que presentan altos porcentajes de población extranjera. Para ello, la investigación se ha centrado en aquellos barrios que, según los datos del Padrón Continuo de 2011, albergaban secciones censales donde el porcentaje de población inmigrante de origen económico era superior al $20 \%$, ya que dicho porcentaje se asocia con el inicio de actitudes xenófobas por parte de la población autóctona (figura 3$)^{12}$. La xenofobia, variable social de evidente carácter subjetivo, fruto del miedo y del desconocimiento de la población autóctona, determina en gran medida el modo en que estos barrios son socialmente percibidos (Iglesias Pascual, 2015). A partir de ello, se estableció la decisión metodológica de orientar el trabajo de campo hacia el análisis del imaginario que, desde el resto de la ciudad, se recrea sobre estos barrios, así como su papel en el desarrollo del filtrado residencial y, por tanto, en el proceso de segregación residencial. Ateniéndonos a este criterio, tres eran en 2011 los ámbitos barriales de Sevilla que albergaban secciones censales con dicho porcentaje de población inmigrante (figura 4): en el distrito 2 (Macarena), los barrios de El Rocío, El Cerezo, La Palmilla-Dr. Marañón, Begoña-Santa Catalina, Las Avenidas y Villegas; en el distrito 4 (CerroAmate), los barrios de Los Pájaros y La Plata; por último, en el distrito 11, el barrio de Los Remedios.

Tal como se ha comentado, los GD y las entrevistas han sido los principales elementos para la recogida de información. Ambas actividades se han realizado en el mismo periodo de tiempo, en concreto, entre septiembre, octubre y la primera quincena de noviembre de 2013. El motivo de acotar todo el trabajo de campo en un periodo tan específico fue el de evitar que cualquier aspecto social o mediático alterase las opiniones de los participantes de manera significativa.

11. Según el Censo de Población de 2011, en el caso de Andalucía, el 62,9\% de sus habitantes vive en zonas metropolitanas.

12. Tal como ha mostrado Herranz de Rafael (2008), la mayor densidad de inmigrantes extranjeros en un ámbito determinado fomenta conductas y actitudes xenófobas de la población autóctona, tanto en el caso de aquellos individuos que viven en municipios pequeños, como en barrios de grandes áreas urbanas. Del mismo modo lo muestran González Enríquez y Álvarez Miranda (2006) en diversos estudios cualitativos centrados en el análisis de estas conductas, utilizando la técnica de las entrevistas mediante grupos de discusión en ciudades como Madrid, Barcelona, Alicante y Valencia. 


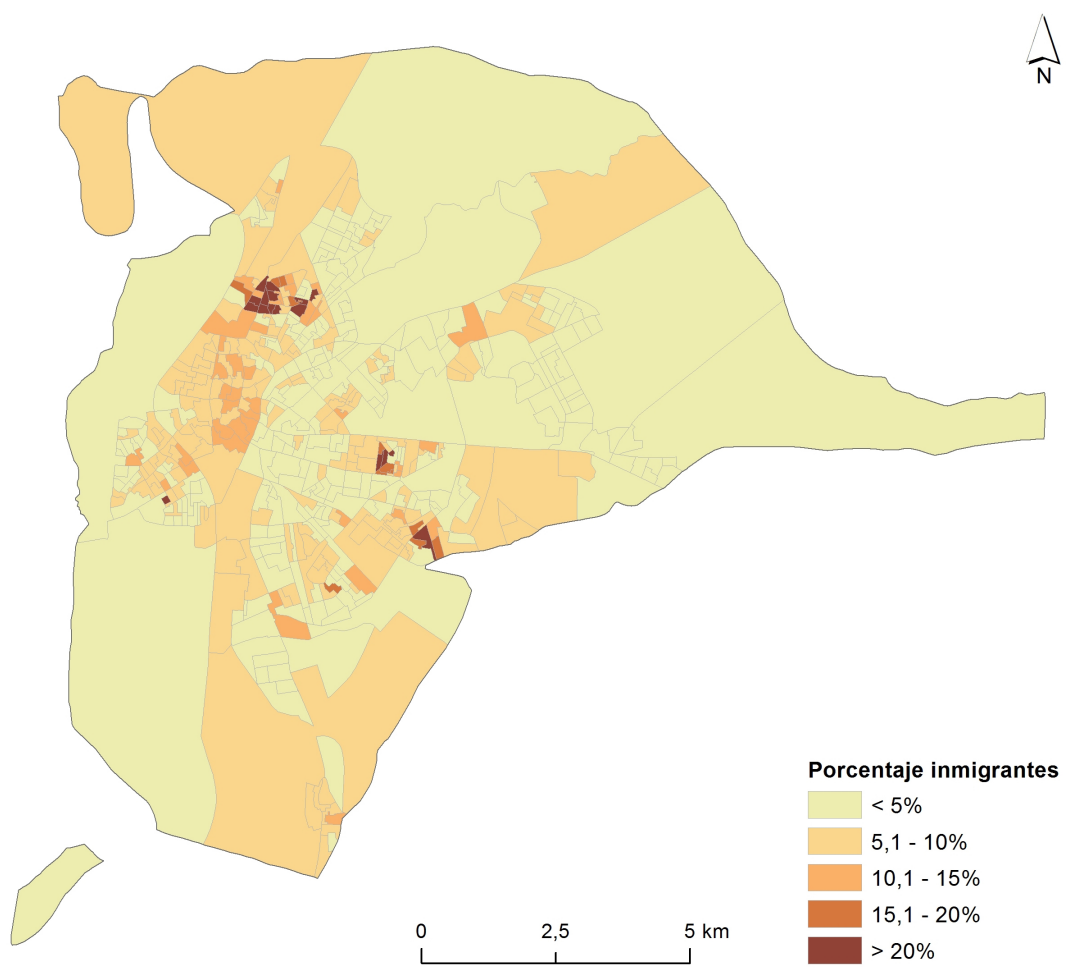

Figura 3. Sevilla. Porcentaje de población inmigrante por secciones censales (2011).

Fuente: SIMA (2011) y elaboración propia.

\subsection{Caracterización y diseño de los grupos de discusión}

A la hora de seleccionar a los participantes en los GD, se buscaron personas que residiesen en zonas exteriores a los ámbitos concretos de estudio, distribuidos en diferentes localizaciones del espacio metropolitano. De este modo, se plantea un análisis de las CES para toda el área metropolitana que permita recoger las variaciones discursivas que en ella se dan según el lugar de residencia. Es decir, se busca valorar qué aspectos de las CES varían a lo largo del área metropolitana y cuáles son comunes en todo el ámbito territorial.

Para conseguir el objetivo de captar la intersubjetividad social del espacio metropolitano y, a su vez, contrastar las diferencias que en él se producen, se desarrollaron un total de ocho GD entre los tres grandes ámbitos establecidos en el espacio metropolitano. Con esta distribución y número de GD, se alcanzó una visión global del discurso que se genera desde el área metropolitana sobre la tipología de barrios que centra la investigación.

A su vez, se consideró relevante realizar un análisis diferenciado de los discursos según el estatus socioeconómico de la población en las diferentes 


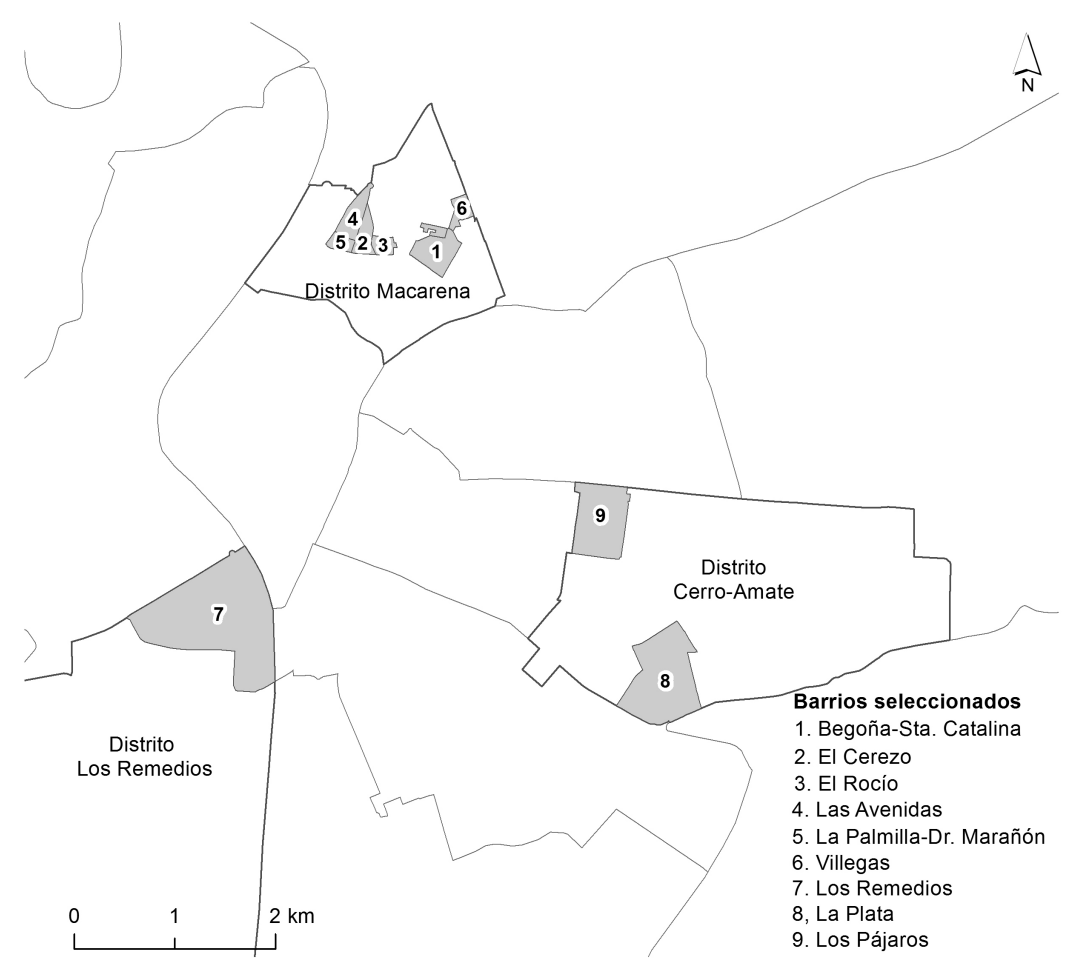

Figura 4. Sevilla. Distritos y barrios que albergan secciones censales con más del $20 \%$ de población inmigrante (2011).

Fuente: elaboración propia.

escalas seleccionadas. Para ello, se establecieron dos grandes estatus sociales, denominados medio-bajo y medio-alto (Tezanos, 2004: 348 s.). El objetivo era determinar la incidencia que la variable socioeconómica mostraba sobre la formación de las CES. Del mismo modo, esta división proporcionaba una muestra de las posiciones sociales de la población autóctona y sus discursos diferenciados respecto a los procesos de aceptación o rechazo de inmigrantes.

Por tanto, para analizar las distintas visiones intersubjetivas y las CES de ellas derivadas, se diseñó un primer conjunto de GD (figura 5) localizados en la ciudad central, cercanos a los barrios determinados como de alta presencia de población extranjera. Así las cosas, se desarrollaron cuatro GD en este primer ámbito. El primero tuvo lugar en el casco histórico (GD1), muy alejado espacial y socialmente respecto a los barrios sobre los que centran la investigación.

Este primer GD se ve acompañado de otros tres. Aunque se encuentran en diferentes zonas de la ciudad consolidada, todos tienen en común que son aledaños a los barrios analizados. De este modo, se organizó un segundo grupo de residentes y exresidentes del barrio de la Macarena (GD 2), un ter- 


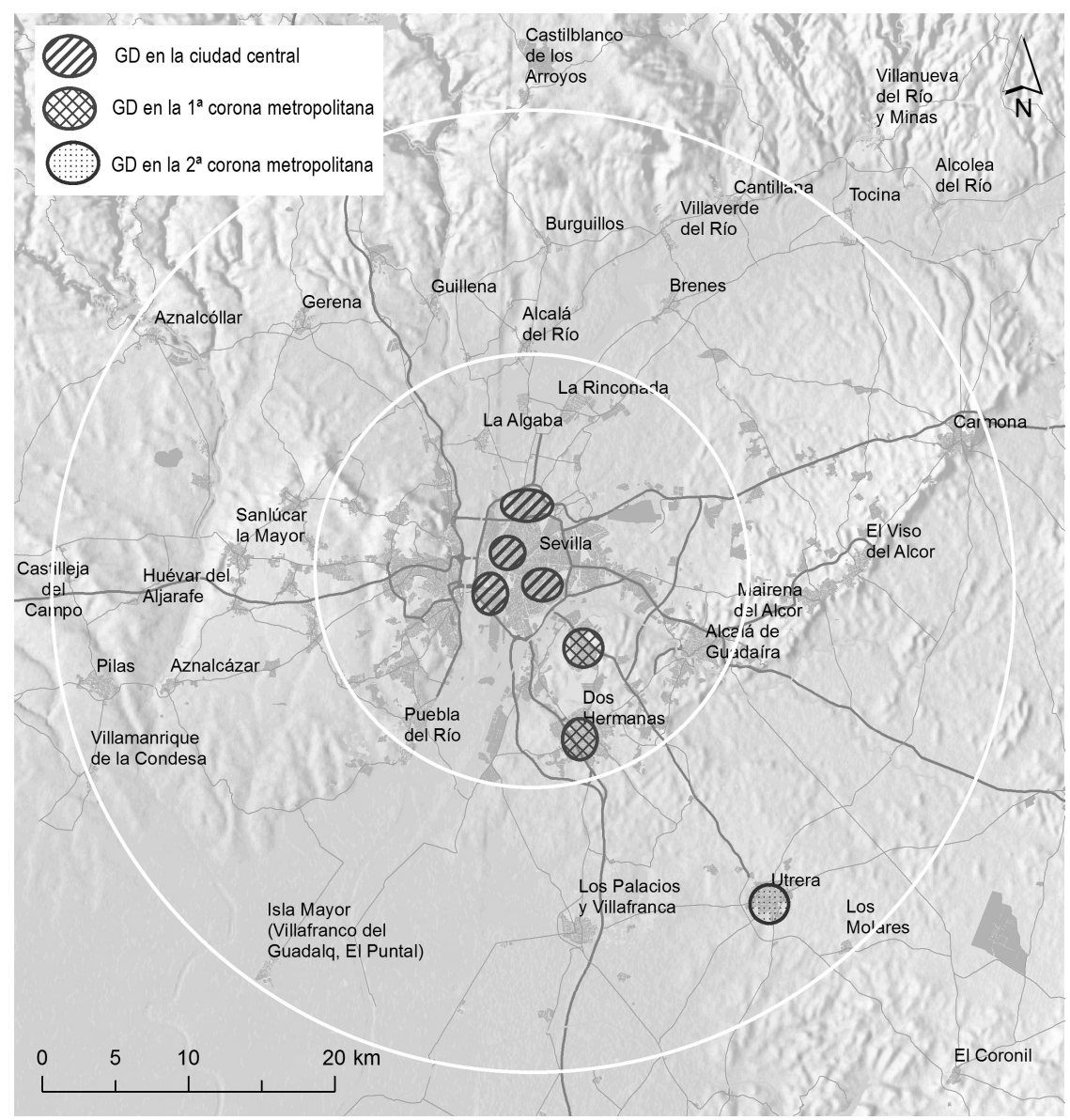

Figura 5. Distribución de los grupos de discusión en el área metropolitana de Sevilla.

Fuente: elaboración propia.

cer grupo de residentes en la zona de Los Remedios (GD 3) y, por último, un cuarto grupo en la zona de Gran Plaza (GD 4). En estos tres últimos casos, se buscaba el discurso de personas cercanas a los barrios pero sin pertenecer estrictamente a ellos, para valorar cómo construyen sus CES, su nivel de convivencia imaginada y real, así como su conocimiento sobre estos espacios.

Para el análisis de las CES en la primera corona metropolitana, se desarrollaron dos grupos. Dentro del estatus socioeconómico medio-bajo, se creó uno de residentes en la barriada de Montequinto (GD 5), en la localidad de Dos Hermanas. Por otro lado, el GD correspondiente al estatus medio-alto estuvo compuesto por cinco vecinos de la localidad de Dos Hermanas y uno de San Juan de Aznalfarache (GD 6), ambos municipios pertenecientes a la 
primera corona. En el caso de la segunda corona metropolitana, los miembros de los grupos realizados en ambos estatus (GD7 y GD8) estuvieron compuestos fundamentalmente por vecinos del municipio de Utrera.

En cuanto a la conducción y al desarrollo de los grupos, siguiendo el criterio de Conde (2009), tras la visualización previa del vídeo, se apostó por una coordinación abierta en su fase inicial, pasando progresivamente a una dirección de carácter más focalizado hacia diferentes aspectos de interés para el estudio. En este sentido, tal como plantean D'Ancona y Valles (2010), tras desarrollarse la discusión libremente en un inicio, se planteaban, en el tramo final de la dinámica, tres cuestiones abiertas al grupo:

— «Estas zonas las conozco por...».

— "La inmigración en la ciudad la asocias a las zonas de...».

— «La marginalidad en la ciudad la asocias a las zonas de...».

Estas frases son consideradas tácticas para incitar la conversación del GD, provocar y observar los discursos sociales sobre inmigración y marginalidad, las posibles interrelaciones que establecían los participantes entre ambas cuestiones y conocer los mecanismos a través de los cuales se construyen las percepciones y los imaginarios sobre la ciudad. En algunos grupos de discusión (GD6 y GD8), estas frases sirvieron para centrar la conversación por primera vez en estas cuestiones ${ }^{13}$, aunque mayoritariamente, al ser temáticas que habían salido antes de manera espontánea, fueron utilizadas de manera general para realizar una simple recapitulación.

\subsection{Profundizando. Las entrevistas}

Tras la realización de los GD diseñados y de un primer análisis general de los discursos generados, se consideró necesario profundizar en algunas cuestiones referentes a las motivaciones que llevaban a los inmigrantes extranjeros a fijar su residencia en distintas zonas de la ciudad. Para ello, se desarrollaron dos entrevistas con un carácter semiestructurado. En ellas, de manera flexible, se buscó indagar sobre las motivaciones y los problemas principales que encuentra la población inmigrante extranjera a la hora de fijar su residencia en la ciudad y, especialmente, sobre las motivaciones que la llevan a concentrarse en determinados barrios de la ciudad. Estas entrevistas fueron realizadas a personas seleccionadas por su amplia experiencia profesional, relacionada con la búsqueda de residencia del colectivo inmigrante. En primer lugar, se entrevistó a la directora de una sucursal de agencia inmobiliaria en un barrio con alta

13. Los grupos de discusión deben ser dinámicas grupales abiertas en las que los participantes hablan libremente y el moderador debe llevar la conversación de una manera implícita a los aspectos que interesan analizar en la investigación, pero nunca de manera clara y abierta. De hacerlo así, no sería un GD, sino un grupo focal o una entrevista grupal. Por esto solo a partir de cierto momento se lanzan estas cuestiones abiertas. Para más detalles sobre diferencia entre grupos de discusión y otras dinámicas grupales, véase Colectivo IOÉ (2010). 
presencia de extranjeros (Macarena). En el segundo caso, la entrevistada fue hecha a una trabajadora social de la CEAR (Confederación Española de Ayuda al Refugiado), responsable de la gestión del servicio de acceso a la vivienda de dicha ONG en Sevilla. Con la información obtenida del análisis de los discursos generados tanto por los GD como por las entrevistas individuales, se consiguió crear una visión general sobre las principales categorías temáticas que forman la base de las CES desarrolladas respecto a estos barrios.

\subsection{La observación participativa. Analizando el doble discurso}

A la importante fuente de información que suponen estos dos primeros instrumentos, se consideró necesario añadir diferentes registros tomados por el investigador sobre las actitudes de los miembros de los GD antes, durante $y$, especialmente, tras la realización de los grupos. El objetivo era registrar el modo cómo los participantes trataban algunos de los temas abordados de una manera más coloquial. Este doble discurso ${ }^{14}$ aporta una importante fuente de información a la hora de valorar el papel de lo implícito y lo oculto en los diferentes discursos individuales. La información así recogida fue especialmente esclarecedora en los grupos correspondientes al estatus medio-alto, donde hay una mayor intención de matizar o encubrir algunos aspectos de sus planteamientos, buscando un discurso "políticamente correcto" (Cea D'Ancona, 2009a: 23). En el caso de los grupos correspondientes al estatus medio-bajo, en pocas ocasiones se observó grandes diferencias entre el discurso público en el grupo de discusión y su posicionamiento posterior.

\subsection{Análisis y transversalidad del discurso}

A la hora de pormenorizar el desarrollo del análisis de los discursos realizados, se deben plantear una serie de cuestiones previas. En primer lugar, es necesario tener en cuenta la importancia de la dimensión territorial en el presente estudio, ya que, además de adentrarnos en los elementos generadores de las CES y su papel en la segregación residencial, se ha considerado de especial importancia analizar las posibles variaciones del discurso según el lugar de residencia en la ciudad y su ámbito metropolitano. Con ello, se buscó valorar la incidencia que adquiere la extensión del ámbito urbano en la construcción, reproducción y permanencia de las CES. Es decir, se pretendía, fundamentalmente, territorializar el imaginario social derivado de los diferentes discursos ciudadanos, seleccionando aquellas temáticas que hacen significativas las variaciones producidas según el lugar de residencia y aquellos constructos sociales que discurren transversalmente a toda la realidad metropolitana.

En este sentido, al abordar el análisis de los discursos generados, se determinó seguir un orden diseñado según la cercanía a las zonas de estudio. De

14. Sobre la dimensión explicita e implícita del discurso, véase, Ruiz (2014) y Martín Criado (2014). 
hecho, Conde (2009) recomienda la lectura y el análisis de los GD siguiendo un orden que permita empezar a establecer comparaciones entre los grupos y sus características locacionales en el espacio metropolitano y en el estatus socioeconómico. Esta lectura ordenada permitió discernir entre las distintas expresividades de cada GD y observar cómo varían sus posiciones según su situación en el espacio metropolitano y en la sociedad. De este modo, se buscaba detectar las principales categorías temáticas socialmente existentes según el lugar ocupado en el espacio metropolitano y su posición socioeconómica. Para ello, en primer lugar, se analizaron los cuatro GD pertenecientes a la ciudad consolidada, los más cercanos a los ámbitos seleccionados. Posteriormente, se analizaron los grupos correspondientes a la primera corona metropolitana, para pasar posteriormente a los de la segunda.

Con esta secuenciación del análisis, se pudo valorar aquellos elementos que se mantienen o cambian a medida que varía la escala de referencia con el objetivo ya citado de plantear el estudio de los discursos que son propios solo a una escala territorial y cuáles son transversales a todo el ámbito metropolitano. Este acercamiento escalar permitió detectar qué categorías temáticas están socialmente generalizadas en todo el espacio metropolitano y cómo varían a lo largo de dicho espacio. La territorialización del imaginario social permitió comprobar cómo se crean, cómo funcionan y, especialmente, cómo se reproducen las CES, aspecto fundamental para entender sus posibles efectos en el inicio o en la acentuación de procesos de segregación residencial asociados a barrios con altas densidades de población extranjera.

En segundo lugar, como se ha dicho con anterioridad, no entraba dentro de los objetivos aquí planteados realizar un análisis sociológico en profundidad de los discursos originados, sino significar los rasgos temáticos comunes, para, de este modo, poder determinar qué arquetipos y percepciones se mantienen en todos los GD, cuáles cambian según el ámbito urbano, así como de qué manera varía la formación de las CES en cada escala del espacio metropolitano. Se buscaba comparar las posiciones ideológicas y el razonamiento principal que están bajo las intersubjetividades de los discursos. Primero de manera individual en cada GD y pasando luego a un análisis intergrupal que permitió realizar una caracterización general de las subjetividades espaciales desarrolladas por la población de Sevilla. En una fase posterior del análisis del discurso, se abordaron aquellas temáticas comunes a todo el territorio metropolitano y que, por lo tanto, se puede considerar que atraviesan transversalmente la ciudad, sirviendo como base fundamental a la hora de entender la construcción de las distintas CES por parte de la ciudadanía.

\section{Consideraciones finales sobre el espacio inducido y el impacto socioterritorial del imaginario sobre la inmigración}

Una vez reflejados los principios epistemológicos que sustentan la propuesta metodológica objeto principal del artículo, la naturaleza de sus instrumentos y el modo de desarrollarlos mediante el trabajo de campo, llega el momento de 
valorar su validez a la hora de analizar la subjetividad socioespacial de manera general y, en concreto, centrada en la escala metropolitana a partir de la propuesta del espacio inducido. De la aplicación de dichos instrumentos, a los que hemos denominado territorialización del discurso, cabe destacar dos aportaciones especialmente relevantes a la hora de determinar qué es y qué implicaciones socioespaciales presenta el espacio inducido. En primer lugar, esta metodología se ha mostrado eficaz a la hora de visibilizar las principales CES desarrolladas sobre los barrios con alta presencia de inmigrantes en Sevilla y cómo varían las categorías discursivas que las componen a lo largo del espacio metropolitano.

Asimismo, también ha permitido determinar el modo en que dichas CES inciden en el proceso de segregación residencial. Es decir, además de explicitar los principales elementos discursivos que contribuyen a recrear intersubjetivamente el imaginario social desarrollado sobre los barrios con altos porcentajes de población inmigrante, permite analizar el papel que la subjetividad espacial ha desempeñado y desempeña en el proceso de segregación residencial de esta población. Es justamente este acercamiento intersubjetivo su principal aportación analítica, puesto que ofrece una alternativa a la metodología tradicionalmente basada en registros individuales que suelen aportar los estudios basados en la percepción.

En cuanto a las principales CES desarrolladas sobre la inmigración y la ciudad, se puede hacer una división en dos grandes categorías. Por un lado, aquellas que muestran aspectos comunes con otros estudios sobre la actitud de la población ante la inmigración y la activación de actitudes xenófobas. En este caso, se trata de subjetividades basadas en la supuesta interrelación entre la presencia de inmigrantes extranjeros y la degradación de los barrios donde residen. En la misma línea destaca la CES relacionada con la visión hipertrofiada del número de inmigrantes en el barrio o la ciudad.

En segundo lugar, cabe reseñar aquellas CES que presentan un carácter más concreto y local, propio de las dinámicas internas de los diferentes barrios de la ciudad de Sevilla. Estas CES se ven determinadas por diferentes factores históricos, populares y socioeconómicos, sin olvidar los estereotipos que, sobre dichos barrios, se han ido desarrollando en la ciudad. Se pueden considerar como opiniones y estereotipos que recrean y retroalimentan la manera en que los ciudadanos del resto de la ciudad miran y actúan respecto a dichos barrios. En este caso, tres han sido las principales CES detectadas: los barrios de la Macarena como personalización de la inmigración en la ciudad ${ }^{15}$; los barrios de Los Pajaritos y Su Eminencia como espacios donde su tradición como zonas marginales de la ciudad (Torres, 2013) «oculta» la creciente presencia de población inmigrante (Iglesias Pascual, 2014a: 331), y la «invisibilidad» del inmigrante en el barrio de Los Remedios, caracterizado en el imaginario

15. Este aspecto está relacionado con que estos barrios fuesen el primer ámbito en el que comenzó a asentarse el colectivo inmigrante a partir de 2001, cuando empieza a residir un número significativo de inmigrantes en la ciudad. Véase Torres et al. (2011) e Iglesias Pascual (2014a: 113 y s.). 
colectivo de la ciudad como ámbito de nivel adquisitivo medio-alto (Torres, 2013; VVAA, 2011). En este caso, resulta evidente que las CES han servido para explicitar las principales zonas de estigmatización territorial de la ciudad (Wacquant, 2007a), así como la dimensión intersubjetiva de su construcción. En este sentido, las CES recreadas sobre los barrios de la Macarena y Los Remedios reflejan claramente el papel que la subjetividad espacial desempeña sobre el proceso de filtrado y segregación residencial. En aquellos barrios de la Macarena que, tal como muestran la CES, son percibidos colectivamente en la ciudad como zonas invadidas por la inmigración, se ha desarrollado un intenso proceso de filtrado residencial desde $2001^{16}$. Sin embargo, en el barrio de Los Remedios, donde el imaginario social colectivo no asocia el barrio con la inmigración, no se ha activado un proceso de filtrado similar, a pesar de albergar una sección censal que en 2011 superaba el 25\% de población inmigrante $^{17}$. Este aspecto sintetiza de manera general el papel que las CES tienen en la movilidad cotidiana y residencial de la población de la ciudad.

En lo referente a la variación territorial de las CES, el análisis ha puesto de manifiesto la transversalidad territorial de diversos elementos temáticos desarrollados sobre los barrios con alta presencia de inmigrantes, destacando especialmente la fuerte influencia del discurso establecido desde la ciudad central. De este modo, se observa cómo los discursos del área central se reflejan y se reproducen de manera generalizada en todo el espacio metropolitano, a pesar del menor conocimiento observado sobre los barrios analizados en las sucesivas coronas metropolitanas. Es decir, las CES que se desarrollan sobre los barrios con alta presencia de inmigrantes son recreadas desde las zonas más cercanas a dichos ámbitos y apropiadas a grandes rasgos por el resto de la ciudad. Esto incide en la formación de lo que hemos denominado espacio inducido, determinando su carácter estereotipado e influyendo en gran medida en la manera de ver y percibir la tipología de estos barrios desde el conjunto del espacio metropolitano. Las CES, por tanto, se crean localmente en puntos concretos de la ciudad, perpetuándose y difundiéndose por todo el área metropolitana como imaginarios sociales que resultan muy difíciles de invertir. Por tanto, se puede hablar de una proyección de las CES de la ciudad consolidada hacia la periferia, interviniendo directamente en la formación del espacio inducido.

Tras el estudio de las diferentes líneas discursivas obtenidas de los GD y de las CES de ellas derivadas, se puede determinar el impacto socioterritorial del imaginario social sobre la inmigración en el área metropolitana de Sevilla, el cual se plasma fundamentalmente en tres variables que reflejan el papel que desempeñan las CES en los procesos de segregación residencial:

- Uno de los efectos principales que se derivan de las CES detectadas es un profundo miedo a la convivencia y el rechazo a la figura del inmigrante. Un

16. Tal como muestran los estudios que han aplicado en la zona diferentes índices de segregación. Véase Huete y Muñoz (2011).

17. Incluso en 2013 ascendió al 32,4\% de población extranjera. 
hipertrofiado sentimiento y percepción de invasión, junto a la asociación que se produce entre inmigración y degradación de los barrios, produce que la población de las sociedades receptoras vea estos ámbitos como espacios peligrosos a evitar. Muestra de ello ha sido la constante caída de la población nacional paralelamente al aumento de la población extranjera en los barrios seleccionados del distrito Macarena ${ }^{18}$.

- La perpetuación de estereotipos relacionados con la inmigración, y, por tanto, la progresiva estigmatización territorial de los barrios, se muestra como otro de los efectos más determinantes del establecimiento de las CES. Resulta evidente tras el análisis de los discursos y sus variaciones en el espacio metropolitano que, cuando se ha establecido socialmente un imaginario colectivo, dicha construcción se transmite sin apenas variación a todo el espacio metropolitano. Esta repetición en el espacio y en los diferentes sectores sociales convierte las CES en estereotipos socioterritoriales que se asientan socialmente sin que ningún tipo de dato objetivo o cuantificable pueda poner en duda los planteamientos que articulan. Su dimensión de construcción colectiva hace que las zonas donde se desarrollan queden caracterizadas de tal modo para toda la ciudad formando parte del espacio inducido colectivo.

- Los dos efectos hasta ahora mencionados se ven cristalizados en la acentuación de las dinámicas situadas en la base del proceso de filtrado residencial y de estigmatización de los barrios en los que se centra el estudio como zonas de escasa o nula deseabilidad residencial. En el caso aquí analizado, las CES influyen sobre los procesos psicosociales que llevan a establecer estos barrios como zonas poco deseables para residir. Partiendo del caso de los barrios indicados en el distrito Macarena, se puede determinar que el proceso de filtrado residencial se inicia cuando el vecindario percibe un incremento en la llegada de individuos de otras nacionalidades.

Finalmente, tras repasar las principales CES que se originan en una ciudad como Sevilla y sus efectos sobre el proceso de segregación residencial, resulta evidente la creciente importancia de abordar el estudio del espacio inducido en el ámbito metropolitano. El conocimiento y la identificación de las CES, su formación, sus dinámicas y sus efectos, se antojan fundamentales a la hora de tomar medidas de intervención político-social para evitar los efectos negativos que la segregación de determinados grupos sociales puede llegar a producir. Un conocimiento cada vez más importante en una sociedad en rápido cambio, caracterizada por sus incertidumbres y la aparición constante de nuevas realidades que hacen dudar al individuo sobre su entorno. Ante este carácter «líquido» de la sociedad urbana, el espacio inducido y las CES que intervienen en su construcción se cosifican socialmente como realidades fundamentales que orientan al individuo en su quehacer diario en la ciudad.

Las características de este espacio inducido hacen necesaria una propuesta metodológica flexible y transdisciplinaria que capte especialmente su dimen- 
sión intersubjetiva. En este sentido, la estrategia metodológica aquí propuesta se puede considerar un instrumento adecuado para determinar cómo el espacio urbano es visto y vivido en sus diferentes escalas. Esta aproximación colectiva, junto a la posibilidad de ir más allá de un análisis meramente descriptivo, se muestra como una de sus principales potencialidades. Aunque en este caso se ha aplicado al análisis de la segregación residencial, también podría hacerse extensible a cuestiones tan variadas como la inseguridad y la delincuencia en la ciudad, los niveles de bienestar y de satisfacción ciudadana, la imagen turística, las necesidades de equipamiento y de infraestructuras urbanas o incluso la imagen colectiva producida por la ciudad desde el punto de vista económico y social. Con esta propuesta metodológica, se pretende aportar nuevas posibilidades al estudio de la dimensión intersubjetiva del espacio urbano, sus diferentes categorizaciones internas, posibilidades de análisis y nuevas realidades.

\section{Referencias bibliográficas}

AguiLAR, Miguel Ángel (2006). «La dimensión estética en la experiencia urbana». En: Lindón, Alicia; Aguilar, Miguel Ángel y Hiernaux, Daniel (coords.). Lugares e imaginarios en la metrópolis. Iztapalapa: Universidad Autónoma Metropolitana. División de Ciencias Sociales y Humanidades. Barcelona: Anthropos.

Alonso, Luis Enrique (1996). «El grupo de discusión en su práctica: Memoria social, intertextualidad y acción comunicativa». Revista Internacional de Sociología, 13, $5-36$.

- (1998). La mirada cualitativa en sociología: Una aproximación interpretativa. Madrid: Fundamentos.

Álvarez Gálvez, Javier (2011). La reproducción mediática del racismo: Diseño de indicadores para la medición de actitudes racistas y xenófobas. Tesis leída en la Universidad Complutense de Madrid.

Augé, Marc (1992). Los no lugares: Espacios del anonimato. Buenos Aires: Gedisa.

Berger, Peter L. y Luckmann, Thomas (1966). The Social Construction of Reality: A Treatise its the Sociology of Knowledge. Nueva York: Anchor Books.

Cea D'Ancona, María Angeles (2004). La activación de la xenofobia en España. Madrid: Centro de Investigaciones Sociológicas.

- (2005). «La exteriorización de la xenofobia». Revista Española de Investigaciones Sociológicas, 112, 197-230.

- (2009a). «La compleja detección del racismo y la xenofobia a través de encuestas: Un paso adelante en su medición». Revista Española de Investigaciones Sociológicas, $125,13-46$.

- (2009b). «Filias y fobias ante la imagen poliédrica cambiante de la inmigración: Claves en la comprensión del racismo y la xenofobia». Revista del Ministerio de Trabajo e Inmigración: Migraciones Internacionales, 80, 39-60.

Cea D’Ancona, María Ángeles y Valles Martínez, Miguel Santiago (2010). Living Together. European Citizenship against racism and xenophobia: Decalogue and final comparative and comprehensive report. Madrid. OBERAXE. Ministerio de Trabajo e Inmigración.

Certeau, Michael de (1980). L'Invention du Quotidien. Volumen 1, Arts de Faire. París: Union générale d'éditions, 10-18. 
Colectivo IOÉ (2010). «Para qué sirve el grupo de discusión: Una revisión crítica del uso de técnicas grupales en los estudios sobre migraciones». Empiria: Revista de Metodología de Ciencias Sociales. Madrid: Universidad Nacional de Educación a Distancia, 19, 73-99.

Conde, Fernando (2007). «Entre al arraigo y la movilidad: La importancia de los imaginarios sociales en la movilidad residencial». En: Feria Toribio, J. M. (coord.). La vivienda y el espacio residencial en las áreas metropolitanas. Sevilla: Centro de Estudios Andaluces. Consejería de Presidencia. Junta de Andalucía.

- (2009). Análisis sociológico del sistema de discursos. Madrid: Centro de Investigaciones Sociológicas.

Corbetta, Perrigorgio (2007). Metodología y técnicas de investigación social. Madrid: McGraw Hill.

Dematteis, Giuseppe (1998). «Suburbanización y periurbanización: Ciudades anglosajonas y ciudades latinas». En: Monclús, F. J. (ed.). La ciudad dispersa. Barcelona: Centre de Cultura Contemporània de Barcelona.

Echazarra, Alfonso (2014). La delincuencia en los barrios: Percepción y reacciones. Barcelona: Obra social 'La Caixa'. Estudios Sociales, 37.

Feria, José María (2013). «Towards a taxonomy of spanish metropolitan areas». Boletín de la Asociación de Geógrafos Españoles, 6, 349-378.

García Canclini, Nestor (1996). "Ciudades y ciudadanos imaginados por los medios». Perfiles Latinoamericanos, 9, 9-24. México: Facultad Latinoamericana de Ciencias Sociales.

- (1997). Imaginarios urbanos. Buenos Aires: Eudeba. Universidad de Buenos Aires.

González EnríQuez, Carmen y Álvarez-Miranda, Berta (2006). Inmigrantes en el barrio: Un estudio cualitativo de opinión pública. Madrid: Ministerio de Trabajo y Asuntos Sociales.

Hall, Peter (1996). Ciudades del mañana: Historia del Urbanismo en el siglo XX. Barcelona: Ediciones del Serbal. La Estrella Polar.

Herranz de Rafael, Gonzalo (2008). «Xenofobia: Un estudio comparativo en barrios y municipios almerienses». CIS, 121, 107-132.

Huete García, María Ángeles y Muñoz Moreno, Rocío (2011). «Modelos de barrio y lógicas de localización de la población inmigrante: El caso de la ciudad de Sevilla». Scripta Nova [en línea], XV (372). Barcelona: Universidad de Barcelona <http://www.ub.es/geocrit/sn/sn-372.htm>.

Hurworth, Rosalind (2003). «Photo-Interviewing for research». Social Research Update [en línea], 40. <http://sru.soc.surrey.ac.uk/SRU40.html>.

IвÁÑEZ, Jesús (1979). Más allá de la sociología. El grupo de discusión: Teoría y crítica. Madrid: Siglo XXI.

IgARTUA, Juan José et al. (2005). «La imagen de la inmigración en la prensa y en la televisión: Aproximaciones empíricas desde la teoría del framing». En: SABUCEdo, J. M.; Romay, J. y López-Cordón, A. Psicología política, cultura, inmigración y comunicación social. Madrid. Biblioteca Nueva, 239-246.

- (2006). «Efectos cognitivos y afectivos de los encuadres noticiosos de la inmigración». En: V Jornadas de Comunicación Medios de Comunicación, Inmigración y Sociedad: Retos y propuestas para el siglo XX. Universidad de Salamanca, 7-9 de marzo de 2006.

Iglesias Pascual, Ricardo (2014a). El papel de las concepciones espaciales subjetivas en la segregación residencial de los barrios con alta densidad de inmigración: El caso de la ciudad de Sevilla [en línea]. Tesis doctoral inédita. Universidad Pablo de Olavide. 
$<$ http://migraciones.ugr.es/cddi/index.php/agradecimientos/article/662-iglesiaspascual-ricardo $>$.

- (2014b). «Generadores del discurso sobre inmigración extranjera y su difusión en el ámbito metropolitano». En: I Simposium Internacional EDiSO 2014. Estudios sobre Discurso y Sociedad [en línea]. Sevilla. <http://www.edisoportal.org/simposium2014/resumenes2014/resumenes2014-seminario-4>.

- (2015). «Segregación residencial, concepciones espaciales subjetivas y xenofobia en Sevilla: Determinando el umbral para la intervención social». Cuadernos Geográficos, 54 (1), 230-256.

InDOVInA, Francesco (1998). "Algunes consideracions sobre la "ciutat difusa"». Documents d'Anàlisi Geogràfica, 33.

Krysan, María (1998). "Privacy and the expression of white racial attitudes: A comparison across three contexts». Public Opinion Quarterly [en línea], 62, 506-544. <http://dx.doi.org/10.1086/297859>

Lindón, Alicia; Aguilar, Miguel Ángel; Hiernaux, Daniel (coords.) (2006). Lugares e imaginarios en la metrópolis. Iztapalapa: Universidad Autónoma Metropolitana. División de Ciencias Sociales y Humanidades. Barcelona: Anthropos.

Lynch, Kevin (1960). The Image of the City. Cambridge: The MIT Press.

Lowenthal, David (1961). "Geography, experience and imagination: Towards a geographical epistemology». Annals of the Association of American Geographers [en línea], 51 (3), 241-260. <http://dx.doi.org/10.1111/j.1467-8306.1961.tb00377.x>

Martín Criado, Enrique (2014). «Mentiras, inconsistencias y ambivalencias: Teoría de la acción y análisis de discurso». Revista Internacional de Sociología [en línea], 72 (1), 115-138. <http://dx.doi.org/10.3989/ris.2012.07.24>

Monclús, Francisco Javier (1998). «Suburbanización y nuevas periferias: Perspectivas geográfico-urbanísticas». En: Monclús, F. J. (ed.). La ciudad dispersa. Barcelona: Centre de Cultura Contemporània de Barcelona.

OrTí, Alfonso (1986). «La apertura y el enfoque cualitativo o estructural: La entrevista abierta semidirectiva y la discusión de grupo». En: García Ferrando, M.; Ibáñez, J.; Alvira, F. (comps.). El análisis de la realidad social: Métodos y técnicas de investigación social. Madrid: Alianza.

Palacios García, Antonio y Vidal Domínguez, María Jesús (2014). «La distribución intraurbana de los inmigrantes en las ciudades españolas: Un análisis de casos con SIG y técnicas cuantitativas». Cuadernos Geográficos, 53 (1), 98-121.

Pintos, Juan Luis (1995). Los imaginarios sociales: La nueva construcción de la realidad social. Santander: Sal Terrae.

Relph, Edward (1976). Place and Placelessness. Londres: Pion.

Rinken, Sebastián y Pérez Yruela, Manuel (2007). Actitudes y opiniones de la población andaluza ante la inmigración. Sevilla: Consejería de Gobernación. Junta de Andalucía.

Ruiz, Jorge (2009). "Análisis sociológico del discurso: Métodos y lógicas». Forum Qualitative Sozialforschung / Forum: Qualitative Social Research [en línea], 10 (2), art. 26. <http://nbn-resolving.de/urn:nbn:de:0114-fqs0902263>.

- (2014). «El discurso implícito: Aportaciones para un análisis sociológico». Revista Española de Investigaciones Sociológicas, 146, abril-junio.

Schutz, Alfred (1972). La fenomenología del mundo social. Buenos Aires: Paidós.

Silva, Armando (1992). Imaginarios urbanos. Bogotá: Tercer Mundo Editores. 
Tezanos, José Felix (2004). La sociedad dividida: Estructuras de clases y desigualdades en las sociedades tecnológicas. Madrid: Biblioteca Nueva.

Torres, Francisco José (2013). Segregación urbana y exclusión social en Sevilla: El paradigma Polígono Sur. Sevilla: Universidad de Sevilla.

Torres, Francisco José et al. (2011). El distrito Macarena de Sevilla: Migraciones recientes y transformaciones urbanas y sociales. Sevilla: Junta de Andalucía. Dirección General de Coordinación de Políticas Migratorias.

Tuan, Yi Fu (1974). Topophilia: A Study of Environmental Perception. Attitudes and Values. Nueva York: Prentice-Hall.

Van Dijk, Teun Adrianus (2003). Racismo y discurso de las élites. Barcelona. Gedisa.

Vilá Valentí, Juan (1983). Introducción al estudio teórico de la geografía. Vol. 1, Objetivos, contenidos y enfoques. Barcelona: Ariel.

VVAA (2011). S. I. Sevilla Imaginada. Edición a cargo de Pedro G. Romero y Armando Silva. Sevilla: Almuzara y UNIA Arte y Pensamiento.

WaCQUANT, Loïc (2007a). Los condenados de la ciudad: Gueto, periferias, Estado. Buenos Aires: Siglo XXI.

- (2007b). "La estigmatización territorial en la edad de la marginalidad avanzada». Ciências Sociais Unisinos, 43 (3), septiembre-diciembre, 193-199. 